\title{
$Q$-Analogues of Clifford and Weyl Algebras-Spinor and Oscillator Representations of Quantum Enveloping Algebras
}

Takahiro Hayashi

Department of Mathematics, Faculty of Science, Nagoya University, Chikusa-ku, Nagoya 464, Japan

\begin{abstract}
We introduce $q$-analogues of Clifford and Weyl algebras. Using these, we construct spinor and oscillator representations of quantum enveloping algebras of type $A_{N-1}, B_{N}, C_{N}, D_{N}$ and $A_{N-1}^{(1)}$. Also we discuss the irreducibility and the unitarity of these representations.
\end{abstract}

\section{Introduction}

Clifford and Weyl algebras are two of the most important algebraic objects in theoretical physics. These algebras represent creation and annihilation of particles satisfying Fermi or Bose statistics. Moreover, they have deep connections with many other important algebras, such as Kac-Moody algebras and Virasoro algebras.

Recently, the progress of the quantum inverse scattering method has led to new algebraic structures known as quantum groups. Jimbo [J1] and Drinfeld [D] defined a one-parameter family of Hopf algebras which can be thought of as a $q$-analogue or quantum deformation of the enveloping algebra of a Kac-Moody algebra (see also [KRS]).

One of the purposes of this paper is to show that the Clifford and Weyl algebras have deformation which is compatible with the deformation of Kac-Moody algebras mentioned above. More precisely, we define $q$-analogues $\mathscr{A}_{q}^{ \pm}$of these algebras and construct algebra homomorphisms from quantum enveloping algebras of type $A_{N}, B_{N}, C_{N}, D_{N}$ and $A_{N}^{(1)}$ to the algebras $\mathscr{A}_{q}^{ \pm}$. Since $\mathscr{A}_{q}^{ \pm}$acts on the exterior or polynomial algebra $V^{ \pm}$as usual, we get spinor and oscillator representations of quantum enveloping algebras.

In Sect. 2, we recall the definition of quantum enveloping algebras and its representation theory briefly. In Sect. 3, we define $q$-analogues $\mathscr{A}_{q}^{ \pm}$of Clifford and Weyl algebras and study representations $V^{ \pm}$which are irreducible if $q$ is not a root of unity. When $q$ is a root of unity, the representation $V^{+}$of $\mathscr{A}_{q}^{+}$ remains irreducible, but the representation $V^{-}$of $\mathscr{A}_{q}^{-}$has countably irreducible components. In Sects. 3 and 4, we construct and study the spinor and oscillator representations of quantum enveloping algebras of type $A_{N-1}, B_{N}, C_{N}$ and $D_{N}$. In 
Sect. 5, the unitarizability of these representations are discussed. In Sect. 6, we construct the spinor and the oscillator representations of quantum enveloping algebras corresponding to the infinite Cartan matrix $A_{\infty}$ and the affine Cartan matrix $A_{N-1}^{(1)}$.

\section{Q-Analogues of the Enveloping Algebras}

1.1 The Hopf Algebra $U_{q}(X)$. Let $X=\left[a_{i j}\right]_{1 \leqq i, j \leqq N}$ be a symmetrizable generalized Cartan matrix and let $d_{i}(1 \leqq i \leqq n)$ be the non-zero integers such that $d_{i} a_{i j}=d_{j} a_{j i}$ and the greatest common divisor of $d_{i}$ 's is 1 . Let $q \neq 0$ be a complex number such that $q^{4 d_{i}} \neq 1$ for any $i$. The quantum enveloping algebra $U_{q}(X)$ corresponding to $X$ and the parameter $q$ is a Hopf algebra with unit 1 and generators $e_{i}, f_{i}, k_{i}^{ \pm 1}$ $(1 \leqq i \leqq N)$ satisfying the following relations:

$$
\begin{gathered}
k_{i} k_{i}^{-1}=k_{i}^{-1} k_{i}=1, \quad k_{i} k_{j}=k_{j} k_{i}, \\
k_{i} e_{j} k_{i}^{-1}=q_{i}^{a_{i j}} e_{j}, \quad k_{i} f_{j} k_{i}^{-1}=q_{i}^{-a_{i j}} f_{j}, \\
e_{i} f_{j}-f_{j} e_{i}=\delta_{i j} \frac{k_{i}^{2}-k_{i}^{-2}}{q_{i}^{2}-q_{i}^{-2}}, \\
\sum_{0 \leqq n \leqq 1-a_{i j}}(-1)^{n}\left[\begin{array}{c}
1-a_{i j} \\
n
\end{array}\right]_{q_{i}^{2}} e_{i}^{1-a_{i j}-n} e_{j} e_{i}^{n}=0 \quad(i \neq j), \\
\Delta\left(e_{i}\right)=e_{i} \otimes k_{i}^{-1}+k_{i} \otimes e_{i}, \quad \Delta\left(f_{i}\right)=f_{i} \otimes k_{i}^{-1}+k_{i} \otimes f_{i}, \quad \Delta\left(k_{i}\right)=k_{i} \otimes k_{i}, \\
\varepsilon\left(e_{i}\right)=0, \quad \varepsilon\left(f_{i}\right)=0, \quad \varepsilon\left(k_{i}\right)=1, \\
S\left(e_{i}\right)=-q_{i}^{-2} e_{i}, \quad S\left(f_{i}\right)=-q_{i}^{2} f_{i}, \quad S\left(k_{i}\right)=k_{i}^{-1},
\end{gathered}
$$

where $\Delta, \varepsilon$ and $S$ denote the coproduct, counit and antipode of $U_{q}(X)$ respectively, $q_{i}=q^{d_{i}}$ and $\left[\begin{array}{c}m \\ n\end{array}\right]_{q}$ is the number defined by

$$
\begin{aligned}
& {\left[\begin{array}{l}
m \\
n
\end{array}\right]_{q}=\frac{[m] !_{q}}{[m-n] !_{q}[n] !_{q}},} \\
& {[m] !_{q}=[m]_{q}[m-1]_{q} \cdots[2]_{q}[1]_{q}, \quad[m]_{q}=\frac{q^{m}-q^{-m}}{q-q^{-1}} .}
\end{aligned}
$$

We call elements $\left\{e_{i}, f_{i}, k_{i} \mid 1 \leqq i \leqq N\right\}$ the Chevalley generators of $U_{q}(X)$. A number of the form $[m]_{q}$ is often called a $q$-integer.

1.2 Representations of $U_{q}(X)$. For $U_{q}(X)$-modules $M$ and $N$, we can define actions of $U_{q}(X)$ on the tensor product $M \otimes N$ and linear dual space $M^{*}$ as follows:

$$
\begin{array}{ll}
a(u \otimes v):=\Delta(a)(u \otimes v), & \left(a \in U_{q}(X), u \in M, v \in N\right), \\
\langle a f, u\rangle:=\langle f, S(a) u\rangle, & \left(a \in U_{q}(X), u \in M, f \in M^{*}\right) .
\end{array}
$$


Let the parameter $q$ not be a root of unity. For a $U_{q}(X)$-module $M$ and $z=\left(z_{1}, \ldots, z_{N}\right) \in\left(\mathbb{C}^{\times}\right)^{N}$, we set $M_{z}=\left\{u \in M \mid k_{i} v=z_{i} v\right\}$. A vector $v \in M$ is said to be primitive if $0 \neq v \in M_{z}$ for some $z \in\left(\mathbb{C}^{\times}\right)^{N}$ and $e_{i} v=0$ for all $i \in\{1, \ldots, N\}$. We say that $M$ is a highest weight module with highest weight $z$, if $M$ is generated by a primitive vector $v \in M_{z}$. For each $z \in\left(\mathbb{C}^{\times}\right)^{N}$, there exists an irreducible highest weight module with highest weight $z$ and it is unique up to isomorphism (see [L1] and [R]).

\section{Q-Analogues of Clifford and Weyl Algebras}

2.1 The Algebras $\mathscr{A}_{q}^{ \pm}$. Let $N$ be a positive integer and $q$ be a non-zero complex number such that $q^{4} \neq 1$. We define algebras $\mathscr{A}_{q}^{ \pm}=\mathscr{A}_{q}^{ \pm}(N)$ with generators $\psi_{i}, \psi_{i}^{\dagger}, \omega_{i}, \omega_{i}^{-1}(1 \leqq i \leqq N)$ by the following defining relations:

$$
\begin{gathered}
\omega_{i} \omega_{j}=\omega_{j} \omega_{i}, \quad \omega_{i} \omega_{i}^{-1}=\omega_{i}^{-1} \omega_{i}=1, \\
\omega_{i} \psi_{j} \omega_{i}^{-1}=q^{ \pm \delta_{i j}} \psi_{j}, \quad \omega_{i} \psi_{j}^{\dagger} \omega_{i}^{-1}=q^{\mp \delta_{i j}} \psi_{j}^{\dagger}, \\
\psi_{i} \psi_{j} \pm \psi_{j} \psi_{i}=\psi_{i}^{\dagger} \psi_{j}^{\dagger} \pm \psi_{j}^{\dagger} \psi_{i}^{\dagger}=0, \\
\psi_{i} \psi_{j}^{\dagger} \pm \psi_{j}^{\dagger} \psi_{i}=0 \quad(i \neq j), \\
\psi_{i} \psi_{i}^{\dagger} \pm q^{2} \psi_{i}^{\dagger} \psi_{i}=\omega_{i}^{-2}, \quad \psi_{i} \psi_{i}^{\dagger} \pm q^{-2} \psi_{i}^{\dagger} \psi_{i}=\omega_{i}^{2} .
\end{gathered}
$$

Note that the relations (2.5) are equivalent to the following relations:

$$
\psi_{i} \psi_{i}^{\dagger}=\frac{\left(q \omega_{i}\right)^{2}-\left(q \omega_{i}\right)^{-2}}{q^{2}-q^{-2}}, \quad \psi_{i}^{\dagger} \psi_{i}=\mp \frac{\omega_{i}^{2}-\omega_{i}^{-2}}{q^{2}-q^{-2}} .
$$

We call the algebras $\mathscr{A}_{q}^{+}$and $\mathscr{A}_{q}^{-}$the q-analogue of Clifford and Weyl algebras (or $q$-Clifford and $q$-Weyl algebras) respectively.

As well as the original case, the algebra $\mathscr{A}_{q}^{+}$(respectively $\mathscr{A}_{q}^{-}$) have a natural representation on exterior (respectively polynomial) algebra in half of the generators. Let $V^{+}$(respectively $V^{-}$) be the exterior (respectively polynomial) algebra generated by elements $x_{i}(1 \leqq i \leqq N)$. For integers $\mathbf{m}=\left(m_{1}, \ldots, m_{N}\right) \in \mathbb{Z}^{N}$, we define an element $x(\mathbf{m})$ of $V^{ \pm}$as follows:

$$
\begin{aligned}
& x(\mathbf{m})=\left\{\begin{array}{cc}
x_{1}^{m_{1}} \wedge x_{2}^{m_{2}} \wedge \cdots \wedge x_{N}^{m_{N}} & \left(\mathbf{m} \in\{0,1\}^{N}\right) \\
0 & (\text { otherwise) }
\end{array}\right)\left(\text { for } \mathscr{A}_{q}^{+}(N)\right), \\
& x(\mathbf{m})=\left\{\begin{array}{cll}
x_{1}^{m_{1}} x_{2}^{m_{2}} \cdots x_{N}^{m_{N}} & \left(\mathbf{m} \in \mathbb{Z}_{\geqq 0}^{N}\right) \\
0 & (\text { otherwise) }
\end{array} \quad\left(\text { for } \mathscr{A}_{q}^{-}(N)\right) .\right.
\end{aligned}
$$

Clearly the set $\left\{x(\mathbf{m}) \mid \mathbf{m} \in\{0,1\}^{N}\right\}$ (respectively $\left\{x(\mathbf{m}) \mid \mathbf{m} \in \mathbb{Z}_{\geq 0}^{N}\right\}$ ) is a basis of the vector space $V^{ \pm}$. Then the action of the algebra $\mathscr{A}_{q}^{ \pm}$on $V^{ \pm}$is given by

$$
\begin{aligned}
& \omega_{i}(x(\mathbf{m}))=q^{-m_{i}} x(\mathbf{m}), \quad \psi_{i}(x(\mathbf{m}))=(-1)^{m_{1}+\cdots+m_{i-1}} x\left(\mathbf{m}-\mathbf{e}_{i}\right), \\
& \psi_{i}^{\dagger}(x(\mathbf{m}))=(-1)^{m_{1}+\cdots+m_{i-1}} x\left(\mathbf{m}+\mathbf{e}_{i}\right), \quad\left(\mathbf{m}=\left(m_{1}, \ldots, m_{N}\right) \in\{0,1\}^{N}\right),
\end{aligned}
$$

for $\mathscr{A}_{q}^{+}$and

$$
\begin{aligned}
& \omega_{i}(x(\mathbf{m}))=q^{m_{i}} x(\mathbf{m}), \quad \psi_{i}(x(\mathbf{m}))=\left[m_{i}\right]_{q^{2}} x\left(\mathbf{m}-\mathbf{e}_{i}\right), \\
& \psi_{i}^{\dagger}(x(\mathbf{m}))=x\left(\mathbf{m}+\mathbf{e}_{i}\right), \quad\left(\mathbf{m}=\left(m_{1}, \ldots, m_{N}\right) \in \mathbb{Z}_{\geqq 0}^{N}\right),
\end{aligned}
$$


for $\mathscr{A}_{q}^{-}$. Here $\mathbf{e}_{i}$ 's are elements of $\mathbb{Z}_{\geqq 0}^{N}$ defined by $\mathbf{e}_{1}=(1,0, \ldots, 0), \ldots, \mathbf{e}_{N}=(0, \ldots, 0,1)$.

Proposition 2.1. (1) The representation $V^{+}$of $\mathscr{A}_{q}^{+}(N)$ is irreducible. (2) If $q$ is not a root of unity, then $V^{-}$is an irreducible representation of $\mathscr{A}_{q}^{-}(N)$.

Proof. Let $W$ be a sub-representation of $V^{-}$and $u=\sum_{\mathbf{m}} a_{\mathbf{m}} x(\mathbf{m})\left(a_{\mathbf{m}} \in \mathbb{C}\right)$ be a non-zero vector of $W$. Let $\mathbf{n}=\left(n_{i}\right)$ be the maximum element of the set $\left\{\mathbf{m} \in \mathbb{Z}_{\geqq 0}^{N} \mid a_{\mathbf{m}} \neq 0\right\}$ with respect to the lexicographic ordering of $\mathbb{Z}_{\geqq 0}^{N}$. Then we have

$$
\left(\psi_{1}\right)^{n_{1}} \cdots\left(\psi_{N}\right)^{n_{N}} u=a_{\mathrm{n}}\left[n_{1}\right] !_{q^{2}} \cdots\left[n_{N}\right] !_{q^{2}} x(\mathbf{0}) .
$$

Since all coefficients $[n]_{q^{2}}$ do not vanish, the vector $x(\mathbf{0})$ belongs to $W$. Now part (2) follows from $V^{-}=\mathscr{A}_{q}^{-}(N) x(\mathbf{0})$. The proof of part (1) is similar.

2.2 The Case $q$ is a Root of 1 . We next consider the case $q^{4}$ is a primitive $L^{\text {th }}$ root of unity. In this case, the representation $V^{-}$is no longer irreducible, since the structure constants $[\mathrm{m}]_{q^{2}}$ may vanish. Here we noted some formulae for $q$-integers:

$$
\begin{aligned}
& {[1]_{q^{2}},[2]_{q^{2}}, \ldots,[L-1]_{q^{2}} \neq 0, \quad[L]_{q^{2}}=0,} \\
& {[m+L p]_{q^{2}}=q^{2 L p}[m]_{q^{2}}, \quad[L-1-m]_{q^{2}}=\left(-q^{2 L}\right)[m+1]_{q^{2}} \quad(m, p \in \mathbb{Z}),}
\end{aligned}
$$

For each $\mathbf{p}=\left(p_{1}, \ldots, p_{N}\right) \in \mathbb{Z}_{\geqq 0}^{N}$, introduce a sub $\mathscr{A}_{q}^{-}$-module $\tilde{V}_{\mathbf{p}}^{-}:=\left(x_{1}^{p_{1}} x_{2}^{p_{2}} \cdots x_{N}^{p_{N}}\right)^{L} V^{-}$ of $V^{-}$, then $\widetilde{V}_{\mathbf{p}}^{-}$is a submodule of $\tilde{V}_{\mathbf{p}^{\prime}}^{-}$if $\mathbf{p}-\mathbf{p}^{\prime} \in \mathbb{Z}_{\geqq 0}^{N}$. Moreover define its quotient $V_{\mathbf{p}}^{-}$by

$$
V_{\mathrm{p}}^{-}:=\tilde{V}_{\mathrm{p}}^{-} / \sum_{i=1}^{N} \tilde{V}_{\mathbf{p}+\mathbf{e}_{i}}^{-}
$$

Denote by $x_{\mathbf{p}}(\mathbf{m})$ the image of the vector $x(\mathbf{m}+L \mathbf{p})$ in $V_{\mathbf{p}}^{-}$. Then $\left\{x_{\mathbf{p}}(\mathbf{m}) \mid \mathbf{m} \in\right.$ $\left.\{0, \ldots, L-1\}^{N}\right\}$ is a basis of $V_{\mathrm{p}}^{-}$and the $\mathscr{A}_{\mathrm{q}}^{-}$-action on $V_{\mathrm{p}}^{-}$is given by

$$
\begin{aligned}
& \omega_{i}\left(x_{\mathbf{p}}(\mathbf{m})\right)=q^{m_{i}+L p_{i}} x_{\mathbf{p}}(\mathbf{m}), \quad \psi_{i}\left(x_{\mathbf{p}}(\mathbf{m})\right)=q^{2 L p_{i}}\left[m_{i}\right]_{q^{2}} x_{\mathbf{p}}\left(\mathbf{m}-\mathbf{e}_{i}\right), \\
& \psi_{i}^{\dagger}\left(x_{\mathbf{p}}(\mathbf{m})\right)=x_{\mathbf{p}}\left(\mathbf{m}+\mathbf{e}_{i}\right), \quad\left(\mathbf{m}=\left(m_{1}, \ldots, m_{N}\right) \in\{0, \ldots, L-1\}^{N}\right) .
\end{aligned}
$$

Proposition 2.2. Suppose $q^{4}$ is a primitive $L^{\text {th }}$ root of unity. Then for $\mathbf{p}=\left(p_{1}, \ldots, p_{N}\right) \in$ $\mathbb{Z}_{\geqq 0}^{N}$, (1) the $\mathscr{A}_{q}^{-}(N)$-module $V_{\mathrm{p}}^{-}$is irreducible. (2) The algebra automorphism $\psi_{i} \mapsto q^{2 L p_{i}} \psi_{i}, \psi_{i}^{\dagger} \mapsto \psi_{i}^{\dagger}, \omega_{i} \mapsto q^{L p_{i}} \omega_{i}$ of $\mathscr{A}_{q}^{-}$carries $V_{\mathrm{p}}^{-}$to the module isomorphic to $V_{0}^{-}$.

Proof. Note $q^{L p_{i}}$ belongs to $\{ \pm 1, \pm \sqrt{-1}\}$, then part (2) is an immediate consequence of the formula (2.14).

Remark. In [M], Morikawa studies another $q$-analogue $W_{N}(q, R)$ of the Weyl algebra over a commutative ring $R$. The algebra $W_{N}(q, R)$ is generated by $a_{i}, \hat{a}_{i}$ $(1 \leqq i \leqq N)$ with defining relations

$$
\begin{aligned}
a_{i} a_{j}-a_{j} a_{i} & =\hat{a}_{i} \hat{a}_{j}-\hat{a}_{j} \hat{a}_{i}=a_{i} \hat{a}_{j}-\hat{a}_{j} a_{i}=0 \quad(i \neq j), \\
\hat{a}_{i} a_{i}-q a_{i} \hat{a}_{i} & =1 .
\end{aligned}
$$

There exists an algebra homomorphism $\imath: W_{N}\left(q^{4}, \mathbb{C}\right) \rightarrow \mathscr{A}_{q}^{-}(N)$ defined by $l\left(a_{i}\right)=$ $\psi_{i}^{\dagger}, l\left(\hat{a}_{i}\right)=\omega_{i}^{2} \psi_{i}(1 \leqq i \leqq N)$. 


\section{Constructions of Representations of Quantum Enveloping Algebras}

\subsection{Identities in $\mathscr{A}_{q}^{ \pm}$}

Lemma 3.1. Let $i, j, k$ be distinct elements of $\{1, \ldots, N\}$ and let $\varphi_{i}$ (respectively $\left.\varphi_{k}\right)$ be either $\psi_{i}$ or $\psi_{i}^{\dagger}\left(\right.$ respectively $\psi_{k}$ or $\left.\psi_{k}^{\dagger}\right)$. Then,

1. the following identities hold in $\mathscr{A}_{q}^{+}$:

$$
\begin{aligned}
& {\left[\psi_{i} \psi_{j}^{\dagger}, \psi_{j} \psi_{i}^{\dagger}\right]=\frac{1}{q^{2}-q^{-2}}\left\{\left(\omega_{i} \omega_{j}^{-1}\right)^{2}-\left(\omega_{i} \omega_{j}^{-1}\right)^{-2}\right\},} \\
& {\left[\psi_{i} \psi_{j}, \psi_{j}^{\dagger} \psi_{i}^{\dagger}\right]=\frac{1}{q^{2}-q^{-2}}\left\{\left(q \omega_{i} \omega_{j}\right)^{2}-\left(q \omega_{i} \omega_{j}\right)^{-2}\right\},} \\
& {\left[\psi_{i}, \psi_{i}^{\dagger}\right]=\frac{1}{q-q^{-1}}\left\{\left(q \omega_{i}^{2}\right)-\left(q \omega_{i}^{2}\right)^{-1}\right\},} \\
& q^{ \pm 2}\left(\varphi_{i} \psi_{j}^{\dagger}\right)\left(\psi_{j} \varphi_{k}\right)-\left(\psi_{j} \varphi_{k}\right)\left(\varphi_{i} \psi_{j}^{\dagger}\right)=\omega_{j}^{\mp 2} \varphi_{i} \varphi_{k}, \\
& q^{ \pm 2}\left(\varphi_{i} \psi_{j}^{\dagger}\right) \psi_{j}-\psi_{j}\left(\varphi_{j} \psi_{j}^{\dagger}\right)=\omega_{j}^{\mp 2} \varphi_{i}, \\
& q^{ \pm 2} \psi_{j}^{\dagger}\left(\psi_{j} \varphi_{k}\right)-\left(\psi_{j} \varphi_{k}\right) \psi_{j}^{\dagger}=\omega_{j}^{\mp 2} \varphi_{k} .
\end{aligned}
$$

2. The following identities hold in $\mathscr{A}_{q}^{-}$:

$$
\begin{aligned}
& {\left[\psi_{i} \psi_{j}^{\dagger}, \psi_{j} \psi_{i}^{\dagger}\right]=\frac{1}{q^{2}-q^{-2}}\left\{\left(\omega_{i}^{-1} \omega_{j}\right)^{2}-\left(\omega_{i}^{-1} \omega_{j}\right)^{-2}\right\},} \\
& {\left[\psi_{i} \psi_{j}, \psi_{j}^{\dagger} \psi_{i}^{\dagger}\right]=\frac{1}{q^{2}-q^{-2}}\left\{\left(q \omega_{i} \omega_{j}\right)^{2}-\left(q \omega_{i} \omega_{j}\right)^{-2}\right\},} \\
& {\left[\left(\psi_{i}\right)^{2},\left(\psi_{i}^{\dagger}\right)^{2}\right]=\frac{q^{2}+q^{-2}}{q^{2}-q^{-2}}\left\{\left(q \omega_{i}^{2}\right)^{2}-\left(q \omega_{i}^{2}\right)^{-2}\right\},} \\
& q^{ \pm 2}\left(\varphi_{i} \psi_{j}^{\dagger}\right)\left(\psi_{j} \varphi_{k}\right)-\left(\psi_{j} \varphi_{k}\right)\left(\varphi_{i} \psi_{j}^{\dagger}\right)=-\omega_{j}^{\mp 2} \varphi_{i} \varphi_{k}, \\
& q^{ \pm 4}\left(\varphi_{i} \psi_{j}^{\dagger}\right)\left(\psi_{j}\right)^{2}-\left(\psi_{j}\right)^{2}\left(\varphi_{i} \psi_{j}^{\dagger}\right)=-\left(q^{2}+q^{-2}\right) \omega_{j}^{\mp 2} \psi_{j} \varphi_{i}, \\
& q^{ \pm 4}\left(\psi_{j}^{\dagger}\right)^{2}\left(\psi_{j} \varphi_{k}\right)-\left(\psi_{j} \varphi_{k}\right)\left(\psi_{j}^{\dagger}\right)^{2}=-\left(q^{2}+q^{-2}\right)\left(q^{-1} \omega_{j}\right)^{\mp 2} \psi_{j}^{\dagger} \varphi_{k} .
\end{aligned}
$$

Proof. Using (2.3) and (2.4), we get,

$$
\left[\psi_{i} \psi_{j}^{\dagger}, \psi_{j} \psi_{i}^{\dagger}\right]=\left(\psi_{i} \psi_{i}^{\dagger}\right)\left(\psi_{j}^{\dagger} \psi_{j}\right)-\left(\psi_{i}^{\dagger} \psi_{i}\right)\left(\psi_{j} \psi_{j}^{\dagger}\right) .
$$

Hence we obtain from (2.6) that

$$
\begin{aligned}
{\left[\psi_{i} \psi_{j}^{\dagger}, \psi_{j} \psi_{i}^{\dagger}\right]=} & \frac{\mp 1}{\left(q^{2}-q^{-2}\right)^{2}}\left\{\left(\left(q \omega_{i}\right)^{2}-\left(q \omega_{i}\right)^{-2}\right)\left(\omega_{j}^{2}-\omega_{j}^{-2}\right)\right. \\
& \left.-\left(\omega_{i}^{2}-\omega_{i}^{-2}\right)\left(\left(q \omega_{j}\right)^{2}-\left(q \omega_{j}\right)^{-2}\right)\right\} \\
= & \frac{1}{q^{2}-q^{-2}}\left(\left(\omega_{i} \omega_{j}^{-1}\right)^{ \pm 2}-\left(\omega_{i} \omega_{j}^{-1}\right)^{\mp 2}\right) .
\end{aligned}
$$


This proves (3.1) and (3.7). The proof of other identities is quite easy and will be omitted.

3.2 Construction of the Representations. Now we will construct representations of quantum enveloping algebras corresponding to Cartan matrices of classical type. Explicitly these matrices are given as follows:

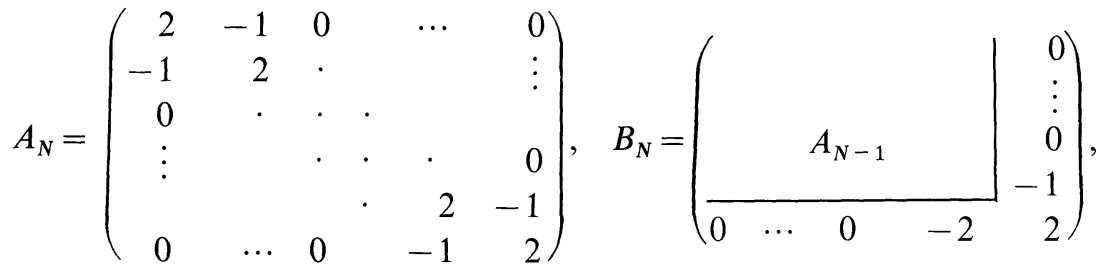



The corresponding non-zero integers $\left(d_{i}\right)=\left(d_{1}, \ldots, d_{N}\right)$ are given by

$$
\begin{array}{llll}
\text { type } A_{N} & \left(d_{i}\right)=(1,1, \ldots, 1), & \text { type } B_{N} & \left(d_{i}\right)=(2, \ldots, 2,1) . \\
\text { type } C_{N} & \left(d_{i}\right)=(1, \ldots, 1,2), & \text { type } D_{N} & \left(d_{i}\right)=(1,1, \ldots, 1) .
\end{array}
$$

Theorem 3.2. There exist algebra homomorphisms defined by the following formulae:

(A)

$$
\begin{gathered}
\pi_{A}^{ \pm}: U_{q}\left(A_{N-1}\right) \rightarrow \mathscr{A}_{q}^{ \pm}(N), \\
e_{i} \mapsto \psi_{i} \psi_{i+1}^{\dagger}, \quad f_{i} \mapsto \psi_{i+1} \psi_{i}^{\dagger}, \quad k_{i} \mapsto\left(\omega_{i} \omega_{i+1}^{-1}\right)^{ \pm 1} \quad(1 \leqq i \leqq N-1),
\end{gathered}
$$

(B)

$$
\begin{array}{lll} 
& \pi_{B}^{+}: U_{q}\left(B_{N}\right) \rightarrow \mathscr{A}_{q^{2}}^{+}(N), \\
e_{i} \mapsto \psi_{i} \psi_{i+1}^{\dagger}, & f_{i} \mapsto \psi_{i+1} \psi_{i}^{\dagger}, & k_{i} \mapsto \omega_{i} \omega_{i+1}^{-1} \quad(1 \leqq i \leqq N-1), \\
e_{N} \mapsto \psi_{N}, & f_{N} \mapsto \psi_{N}^{\dagger}, & k_{N} \mapsto q \omega_{N} .
\end{array}
$$

(C)

$$
\pi_{C}^{-}: U_{q}\left(C_{N}\right) \rightarrow \mathscr{A}_{q}^{-}(N),
$$

$$
\begin{array}{lll}
e_{i} \mapsto \psi_{i} \psi_{i+1}^{\dagger}, & f_{i} \mapsto \psi_{i+1} \psi_{i}^{\dagger}, & k_{i} \mapsto \omega_{i}^{-1} \omega_{i+1} \quad(1 \leqq i \leqq N-1), \\
e_{N} \mapsto \frac{1}{q^{2}+q^{-2}}\left(\psi_{N}\right)^{2}, & f_{N} \mapsto \frac{-1}{q^{2}+q^{-2}}\left(\psi_{N}^{\dagger}\right)^{2}, & k_{N} \mapsto q^{-1} \omega_{N}^{-2} .
\end{array}
$$

(D)

$$
\begin{aligned}
& \pi_{D}^{+}: U_{q}\left(D_{N}\right) \rightarrow \mathscr{A}_{q}^{+}(N), \\
& e_{i} \mapsto \psi_{i} \psi_{i+1}^{\dagger}, \quad f_{i} \mapsto \psi_{i+1} \psi_{i}^{\dagger}, \quad k_{i} \mapsto \omega_{i} \omega_{i+1}^{-1} \quad(1 \leqq i \leqq N-1), \\
& e_{N} \mapsto \psi_{N-1} \psi_{N}, \quad f_{N} \mapsto \psi_{N}^{\dagger} \psi_{N-1}^{\dagger}, \quad k_{N} \mapsto q \omega_{N-1} \omega_{N} .
\end{aligned}
$$

Proof. This is an immediate consequence of the defining relations of the algebras $\mathscr{A}_{q}^{ \pm}$and the above lemma. Here we give the proof only for $\pi_{A}^{-}$. Define elements 
$\tilde{e}_{i}, \tilde{f}_{i}, \tilde{k}_{i}$ of $\mathscr{A}_{q}^{-}$by the right-hand sides of the arrows of the formula (3.17). It suffices to show that these elements satisfy the relations (1.1)-(1.5) for $X=A_{N-1}$. The relations (1.1), (1.2) and (1.3) for $i=j$ directly follow from (2.1), (2.2) and (3.7). For $i \neq j$, (1.3) easily follows from (2.3) and (2.4). If $|i-j| \geqq 2$, then (1.4) follows easily from (2.3), (2.4). Suppose $j=i+1$. Then the right-hand side of (1.4) equals

$$
\begin{aligned}
\tilde{e}_{i} \tilde{e}_{i} \tilde{e}_{i+1}-\left(q^{2}+q^{-2}\right) \tilde{e}_{i} \tilde{e}_{i+1} \tilde{e}_{i}+\tilde{e}_{i+1} \tilde{e}_{i} \tilde{e}_{i}= & \tilde{e}_{i}\left(\tilde{e}_{i} \tilde{e}_{i+1}-q^{2} \tilde{e}_{i+1} \tilde{e}_{i}\right) \\
& +q^{-2}\left(\tilde{e}_{i} \tilde{e}_{i+1}-q^{2} \tilde{e}_{i+1} \tilde{e}_{i}\right) \tilde{e}_{i} .
\end{aligned}
$$

By (3.10), we get

$$
\tilde{e}_{i} \tilde{e}_{i+1}-q^{2} \tilde{e}_{i+1} \tilde{e}_{i}=-q^{2} \omega_{i+1}^{2} \psi_{i} \psi_{i+2}^{\dagger} .
$$

Substituting this to the right-hand side of (3.21), and using (2.2), (2.3) and (2.4), the left-hand side of (3.21) proves to vanish, that is, the formula (1.4) holds for $j=i+1$. The case for $j=i-1$ is similar.

Now we get an action $\rho_{X}^{ \pm}$of the quantum enveloping algebra $U_{q}(X)$ on $V^{ \pm}$through the homomorphism $\pi_{X}^{ \pm}$of this theorem.

Definition. For $X=A_{N-1}, B_{N}, D_{N}$, we call $\rho_{X}^{+}$the spinor representation $U_{q}(X)$ on $V^{+}$. For $X=A_{N-1}, C_{N}$, we call $\rho_{X}^{-}$the oscillator representation of $U_{q}(X)$ on $V^{-}$.

\section{Structure of the Spinor and the Oscillator Representations}

4.1 Generic Case. Now we proceed to study the spinor and the oscillator representations. It is easy to see that $V^{ \pm}$has the following direct sum decomposition as a $U_{q}\left(A_{N-1}\right)$-module:

$$
V^{ \pm}=\bigoplus_{r=0}^{\infty} V_{r}^{ \pm}, \quad V_{r}^{ \pm}=\bigoplus_{|\mathbf{m}|=r} \mathbb{C} x(\mathbf{m}) .
$$

Here for $\mathbf{m}=\left(m_{i}\right) \in \mathbb{Z}_{\geqq 0}^{N}$, we set $|\mathbf{m}|=\sum_{i} m_{i}$. Note that $V_{r}^{+}=0$ for $r>N$. Similarly, as a $U_{q}\left(C_{N}\right)$-(respectively $U_{q}\left(D_{N}\right)$-)module, $V^{-}$(respectively $\left.V^{+}\right)$is decomposed as follows:

$$
V^{ \pm}=V_{\text {even }}^{ \pm} \oplus V_{\text {odd }}^{ \pm}, \quad V_{\text {even }}^{ \pm}=\bigoplus_{r=0}^{\infty} V_{2 r}^{ \pm}, \quad V_{\text {odd }}^{ \pm}=\bigoplus_{r=0}^{\infty} V_{2 r+1}^{ \pm} .
$$

We will first consider the case where the parameter $q$ is not a root of unity. The following theorem follows from standard arguments (see e.g. $[\mathrm{L} 1, \mathrm{R}]$ ).

Theorem 4.1. Let $q$ not be a root of unity. Then we have,

(A) The $U_{q}\left(A_{N-1}\right)$-module $V_{r}^{+}(0 \leqq r \leqq N)$ (respectively $\left.V_{r}^{-}\left(r \in \mathbb{Z}_{\geqq 0}\right)\right)$ is irreducible with highest weight vector $x\left(\mathbf{e}_{N-r+1}+\cdots+\mathbf{e}_{N}\right)$ (respectively $\left.x\left(\mathbf{r e}_{N}\right)\right)$ of weight $\left(q^{\delta_{N-r, i}}\right)_{1 \leqq i \leqq N-1}\left(\right.$ respectively $\left.\left(1, \ldots, 1, q^{r}\right)\right)$.

(B) The $U_{q}\left(B_{N}\right)$-module $V^{+}$is irreducible with highest weight vector $x(\mathbf{0})$ of weight $(1, \ldots, 1, q)$.

(C) The $U_{q}\left(C_{N}\right)$-module $V_{\text {even }}^{-}$(respectively $\left.V_{\text {odd }}^{-}\right)$is irreducible with highest weight vector $x(\mathbf{0})\left(\right.$ respectively $\left.x\left(\mathbf{e}_{N}\right)\right)$ of weight $\left(1, \ldots, 1, q^{-1}\right)\left(\right.$ respectively $\left.\left(1, \ldots, 1, q, q^{-3}\right)\right)$. 
(D) The $U_{q}\left(D_{N}\right)$-module $V_{\text {even }}^{+}\left(\right.$respectively $\left.V_{\text {odd }}^{+}\right)$is irreducible with highest weight vector $x(\mathbf{0})$ (respectively $x\left(\mathbf{e}_{N}\right)$ ) of weight $(1, \ldots, 1, q)$ (respectively $(1, \ldots, 1, q, 1)$ ).

Now consider the representation $V^{+}$for a root $q$ of unity.

Theorem 4.2. The representations $V_{r}^{+}$of $U_{q}\left(A_{N-1}\right)$ (respectively $V_{\text {even }}^{+}$and $V_{\text {odd }}^{+}$of $U_{q}\left(D_{N}\right), V^{+}$of $\left.U_{q}\left(B_{N}\right)\right)$ are irreducible for any $q\left(q^{4} \neq 1\right.$ for $X=A_{N-1}$ and $D_{N} ; q^{8} \neq 1$ for $X=B_{N}$ ).

Proof. Since the matrix coefficients of the representation of $\psi_{i}$ and $\psi_{i}^{\dagger}$ do not depend on the parameter $q$, those of the Chevalley generators $e_{i}$ 's and $f_{i}$ 's also do not depend on $q$. It is easy to check that the Lie subalgebra of End $V^{+}$generated by $e_{i}$ 's and $f_{i}$ 's is isomorphic to $\mathfrak{s l}(N, \mathbb{C}), \mathfrak{s o}(2 N+1)$ or $\mathfrak{s o}(2 N)$. Hence the result follows from the original theory of spinor representations.

4.2 Degenerate Case. Let $q^{4}$ be a primitive $L^{\text {th }}$ root of 1 . For $r \in \mathbb{Z}_{\geqq 0}$ and $\mathbf{p} \in \mathbb{Z}_{\geqq 0}^{N}$, we define a sub $U_{q}\left(A_{N-1}\right)$-module $V_{\mathrm{p}, r}^{-}$of $V_{\mathrm{p}}^{-}$to be the image of $V_{r}^{-}$ by the surjection $V^{-} \rightarrow V_{\mathrm{p}}^{-}$. It is easy to see that $V_{\mathrm{p}, r}^{-} \neq 0$ if and only if $L|\mathbf{p}| \leqq r \leqq L|\mathbf{p}|+(L-1) N$. For $0 \leqq r \leqq(L-1) N$, we set $K_{r}=V_{\mathbf{0}, r}^{-}$.

Theorem 4.3. (1) The $U_{q}\left(A_{N-1}\right)$-module $V_{\mathrm{p}, r}^{-}$is irreducible and generated by the vector $v_{\mathbf{p}, r}:=x_{\mathbf{p}}\left(\mathbf{e}_{N-s}+(L-1)\left(\mathbf{e}_{N-s+1}+\cdots+\mathbf{e}_{N}\right)\right)$, where integers $s$ and $t$ are defined by $r=(L-1) s+t, 0 \leqq t<L-1$.

(2) The algebra automorphism $e_{i} \mapsto q^{2 L p_{i}} e_{i}, f_{i} \mapsto q^{2 L p_{i}+1} f_{i}, \quad k_{i} \mapsto q^{L\left(p_{i+1}-p_{\imath}\right)} k_{i}$ of $U_{q}\left(A_{N-1}\right)$ carries $V_{\mathbf{p}, r}^{-}$to the module isomorphic to $K_{r-|\mathbf{p}|}$.

Proof. Part (2) follows easily from (2.14). Hence it is sufficient to show (1) for $\mathbf{p}=\mathbf{0}$. Let $u=\sum_{|\mathbf{n}|=\mathbf{r}} a_{\mathbf{n}} x_{\mathbf{0}}(\mathbf{n})$ be a non-zero element of a sub-module of $K_{r}$ and let $\mathbf{m}=\left(m_{i}\right)$ be the maximum element of the set $\left\{\mathbf{n} \mid a_{\mathbf{n}} \neq 0\right\}$ with respect to the lexicographic ordering $\leqq$ of $\mathbb{Z}_{\geqq 0}^{N}$. We will show that there exists an element $E$ of $\operatorname{Im} \pi_{\boldsymbol{A}}^{-}$such that

$$
\operatorname{Ex}_{\mathbf{0}}(\mathbf{n})= \begin{cases}C v_{0, r} & (\mathbf{n}=\mathbf{m}) \\ 0 & (\mathbf{n}<\mathbf{m},|\mathbf{n}|=r)\end{cases}
$$

for some $C \neq 0$. Using (3.10), we see that the following elements belong to the image of $\pi_{A}^{-}$:

$$
\begin{aligned}
& e_{i j}:=\omega_{i+1}^{2} \omega_{i+2}^{2} \cdots \omega_{j-1}^{2} \psi_{i} \psi_{j}^{\dagger}, \\
& e_{j i}:=\omega_{i+1}^{2} \omega_{i+2}^{2} \cdots \omega_{j-1}^{2} \psi_{j} \psi_{i}^{\dagger}, \quad(1 \leqq i<j \leqq N) .
\end{aligned}
$$

Set $i(\mathbf{m}):=\min \left\{i \mid m_{i} \neq 0\right\}$ and $j(m):=\max \left\{j \mid \mathbf{m}_{j} \neq L-1\right\}$. Then we see that $i(\mathbf{m}) \leqq j(\mathbf{m})+1$ and that $i(\mathbf{m}) \geqq j(\mathbf{m})$ if and only if $\mathbf{m}=t \mathbf{e}_{N-s}+(L-1)\left(\mathbf{e}_{N-s+1}+\cdots+\right.$ $\left.\mathbf{e}_{N}\right)$. If $i(\mathbf{m})<j(\mathbf{m})$, then we set $\rho(\mathbf{m})=\mathbf{m}-\mathbf{e}_{i(\mathbf{m})}+\mathbf{e}_{j(\mathbf{m})}$. Clearly we have

$$
\begin{gathered}
i(\mathbf{m}) \leqq i(\rho(\mathbf{m})), \quad j(\rho(\mathbf{m})) \leqq j(\mathbf{m}), \\
e_{l(\mathbf{m}) j(\mathbf{m})} x_{0}(\mathbf{m})=c x_{0}(\rho(\mathbf{m})), \quad e_{j(\mathbf{m})(\mathbf{m})} x_{0}(\rho(\mathbf{m}))=c^{\prime} x_{0}(\mathbf{m})
\end{gathered}
$$

for some $c, c^{\prime} \neq 0$. It is easy to see that we can determine sequences $\mathbf{m}=\mathbf{m}_{0}, \mathbf{m}_{1}, \ldots$, $\mathbf{m}_{\mu} \in\{0, \ldots, L-1\}^{N}, i_{0}, \ldots, i_{\mu}, j_{0}, \ldots, j_{\mu} \in\{1, \ldots, N\}$ uniquely by the following condi- 
tions:

$$
\mathbf{m}_{v+1}=\rho\left(\mathbf{m}_{v}\right), \quad i_{v}=i\left(\mathbf{m}_{v}\right), \quad j_{v}=j\left(\mathbf{m}_{v}\right), \quad i_{0}<j_{0}, \quad i_{1}<j_{1}, \ldots, i_{\mu-1}<j_{\mu-1}, \quad i_{\mu} \geqq j_{\mu} .
$$

Then we have $x_{0}\left(\mathbf{m}_{k}\right)=v_{0, r}$ and

$$
\begin{aligned}
E x_{0}(\mathbf{m}) & =C v_{0, r}, \quad F v_{0, r}=C^{\prime} x_{0}(\mathbf{m}), \\
E & =e_{i_{\mu-1} j_{\mu-1}} \cdots e_{i_{1} j_{1}}, \quad F=e_{j_{1} i_{1}} \cdots e_{j_{\mu-1} i_{\mu-1}}
\end{aligned}
$$

for some $C, C^{\prime} \neq 0$. Moreover, since card $\left\{v \mid 1 \leqq v \leqq \mu, i_{v}=k\right\}=m_{k}$ for $1 \leqq k \leqq N-s$ and card $\left\{v \mid 1 \leqq v \leqq \mu, j_{v}=k\right\}=L-1-m_{k}$ for $N-s+1 \leqq k \leqq N$, we have $\operatorname{Ex}_{0}(\mathbf{n})=0$ for any element $\mathbf{n}<\mathbf{m}$ such that $|\mathbf{n}|=r$. Thus we get $v_{0, r} \in W$. On the other hand, by (4.7), we get $U_{q}\left(A_{N-1}\right) v_{0, r}=K_{r}$ and the theorem is proved.

Proposition 4.4. (1) The algebra automorphism $e_{i} \mapsto q^{2 L-2} e_{i}, f_{i} \mapsto q^{2 L+2} f_{i}, k_{i} \mapsto k_{i}$ carries the dual module $\left(K_{r}\right)^{*}$ to the module isomorphic to $K_{(L-1) N-r}$.

(2) The dimensions of the module $K_{r}$ is given by

$$
\operatorname{dim} K_{r}=\sum_{k=0}^{N}(-1)^{k}\left(\begin{array}{c}
N \\
k
\end{array}\right) \cdot\left(\begin{array}{c}
N+r-1-L k \\
N-1
\end{array}\right) .
$$

Proof. Part (1) follows from direct computation. To prove part (2), we introduce symmetric functions $h_{r}$ and $h_{r}^{L}$ by

$$
\begin{aligned}
\sum_{r=0}^{\infty} h_{r}^{L}\left(z_{1}, \ldots, z_{N}\right) t^{r} & =\prod_{i=1}^{N}\left(1+z_{i} t+\cdots+\left(z_{i} t\right)^{L-1}\right), \\
\sum_{r=0}^{\infty} h_{r}\left(z_{1}, \ldots, z_{N}\right) t^{r} & =\prod_{i=1}^{N}\left(1+z_{i} t+\left(z_{i} t\right)^{2}+\cdots\right) .
\end{aligned}
$$

The monomial $z_{1}^{m_{1}} \cdots z_{N}^{m_{N}}$ occurs in $h_{r}^{L}$ with coefficient 1 if $\mathbf{m}=\left(m_{i}\right) \in\{0, \ldots, L-1\}^{N}$ and $|\mathbf{m}|=r$, and does not occur if otherwise. Hence it suffices to calculate $h_{r}^{L}(1, \ldots, 1)$. Let $\left\langle c_{i}\right\rangle(1 \leqq i \leqq N)$ be a cyclic group of order $L$ with a generator $c_{i}$. Then, in $\mathbb{C}\left[\left\langle c_{1}\right\rangle \times \cdots \times\left\langle c_{N}\right\rangle\right] \otimes \mathbb{C}[[t]]$, we have

$$
\begin{aligned}
\sum_{r=0}^{\infty} h_{r}\left(c_{1}, \ldots, c_{N}\right) t^{r} & =\prod_{i=1}^{N}\left(1+c_{i} t+\cdots+\left(c_{i} t\right)^{L-1}\right)\left(1+t^{L}+t^{2 L}+\cdots\right) \\
& =\left(1-t^{L}\right)^{-N} \sum_{r=0}^{\infty} h_{r}^{L}\left(c_{1}, \ldots, c_{N}\right) t^{r} .
\end{aligned}
$$

Using this, we get

$$
h_{r}^{L}\left(c_{1}, \ldots, c_{N}\right)=\sum_{k=0}^{N}(-1)^{k}\left(\begin{array}{l}
N \\
k
\end{array}\right) h_{r-L k}\left(c_{1}, \ldots, c_{N}\right) .
$$

Since $h_{r}(1, \ldots, 1)=\operatorname{dim}\left(V_{r}^{-}\right)=\left(\begin{array}{c}N+r-1 \\ r\end{array}\right)$, we get the desired result.

Next we consider the action of $U_{q}\left(C_{N}\right)$ on $V_{\mathrm{p}}^{-}$. We define submodules $V_{\mathrm{p} \text {,even }}^{-}$ 
$V_{\mathrm{p}, \mathrm{odd}}^{-}$by

$$
V_{\mathrm{p}, \mathrm{even}}^{-}=\bigoplus_{r \geqq 0} V_{\mathrm{p}, 2 r}^{-}, \quad V_{\mathrm{p}, \mathrm{odd}}^{-}=\bigoplus_{r \geqq 0} V_{\mathrm{p}, 2 r+1}^{-} .
$$

Similarly as Proposition 2.1, we get,

Theorem 4.5. (1) The $U_{q}\left(C_{N}\right)$-modules $V_{\mathrm{p} \text {,even }}^{-}$and $V_{\mathrm{p} \text {,odd }}^{-}$are irreducible and generated by the vectors $x_{\mathbf{p}}(\mathbf{0})$ and $x_{\mathbf{p}}\left(\mathbf{e}_{N}\right)$ respectively.

(2) The following algebra automorphism carries $V_{\mathrm{p}, \text { even }}^{-}$and $V_{\mathrm{p}, \text { odd }}^{-}$to the modules isomorphic to $V_{0, \text { even }}^{-}$and $V_{0, \text { odd }}^{-}$respectively.

$$
\begin{aligned}
& e_{i} \mapsto q^{2 L p_{i}} e_{i}, \quad f_{i} \mapsto q^{2 L p_{i}+1} f_{i}, \quad k_{i} \mapsto q^{L\left(p_{i+1}-p_{i}\right)} k_{i} \quad(1 \leqq i<N), \\
& e_{N} \mapsto e_{N}, \quad f_{N} \mapsto f_{N}, \quad k_{N} \mapsto q^{2 L p_{N}} k_{N} .
\end{aligned}
$$

(3) If $L$ is even then $\operatorname{dim} V_{\mathrm{p}, \text { even }}^{-}=\operatorname{dim} V_{\mathrm{p}, \mathrm{odd}}^{-}=L^{N} / 2$. If $L$ is odd then $\operatorname{dim} V_{\mathrm{p}, \text { even }}^{-}=$ $\left(L^{N}+1\right) / 2$ and $\operatorname{dim} V_{\mathrm{p}, \text { odd }}^{-}=\left(L^{N}-1\right) / 2$.

\section{Unitarity of the Representations}

In this section, we will discuss the unitarizability of the representations constructed in the previous sections. Throughout this section, we deal only the case the parameter $q$ belongs to the set $\mathbb{R}^{\prime}:=\mathbb{R} \backslash\{ \pm 1\}$ or the set $\mathbb{T}^{\prime}:=\{z \in \mathbb{C}|| z \mid=1, z \neq \pm 1$, $\pm \sqrt{-1}\}$.

5.1. *-Algebras. First we prepare some terminology. Let $A$ be an algebra over the complex field $\mathbb{C}$. We say that $A$ is a $*$-algebra if there exists an anti-linear, anti-algebra automorphism $*: a \mapsto a^{*}(a \in A)$ such that $\left(a^{*}\right)^{*}=a$ for all $a \in A$. The map $*$ is called the *-operation of $A$. If an algebra map $f$ from $A$ to another *-algebra preserves the *-operation, then $f$ is called a *-homomorphism. A representation $W$ of $A$ is called a *-representation if there exists a Hermitian form (|) such that $(\mathrm{au} \mid v)=\left(u \mid\left(a^{*}\right) v\right)$ for every $u, v \in W$ and $a \in A$ If, in addition, (|) is positive definite, then $W$ is called a unitary representation of the $*$-algebra $A$.

5.2. A*-Operation on $\mathscr{A}_{q}^{ \pm}$. Now we define a $*$-operation on $\mathscr{A}_{q}^{ \pm}(N)$ by the following formulae:

$$
\left(\psi_{i}\right)^{*}=\psi_{i}^{\dagger}, \quad\left(\psi_{i}^{\dagger}\right)^{*}=\psi_{i}, \quad\left(\omega_{i}\right)^{*}=\left\{\begin{array}{ll}
\omega_{i} & \left(q \in \mathbb{R}^{\prime}\right) \\
\omega_{i}^{-1} & \left(q \in \mathbb{T}^{\prime}\right)
\end{array} .\right.
$$

We can determine unitarizability of the representations of $\mathscr{A}_{q}^{ \pm}$constructed in the previous sections.

Proposition 5.1. The module $V^{+}$has a unique Hermitian form (|) such that $(x(0) \mid x(\mathbf{0}))=1$ and $\left(V^{+},(\mid)\right)$is a unitary representation of $\mathscr{A}_{q}^{+}$for all $q \in \mathbb{R}^{\prime}$ or $\mathbb{T}^{\prime}$. Explicitly the form $(\mid)$ is given by

$$
(x(\mathbf{m}) \mid x(\mathbf{n}))=\delta_{\mathbf{m}, \mathbf{n}} \quad\left(\mathbf{m}, \mathbf{n} \in\{0,1\}^{N}\right) .
$$

Proof. It is easy to verify that the module $V^{+}$is a unitary representation with 
respect to the Hermitian form (5.2). The uniqueness of the form follows from $V^{+}=\mathscr{A}_{q}^{+}(N) x(\mathbf{0})$.

Proposition 5.2. The module $V^{-}$has a unique Hermitian form (|) such that $(x(\mathbf{0}) \mid x(\mathbf{0}))=1$ and $\left(V^{-},(\mid)\right)$is a $*$-representation of $\mathscr{A}_{q}^{-}$. Explicitly the form $(\mid)$is given by

$$
(x(\mathbf{m}) \mid x(\mathbf{n}))=\delta_{\mathbf{m}, \mathbf{n}}\left[m_{1}\right] !_{q^{2}}\left[m_{2}\right] !_{q^{2}} \cdots\left[m_{N}\right] !_{q^{2}} \quad\left(\mathbf{m}, \mathbf{n} \in \mathbb{Z}_{\geqq 00}^{N}\right) .
$$

The representation $\left(V^{-},(\mid)\right)$is unitary for $q \in \mathbb{R}^{\prime}$, but is not unitary for $q \in \mathbb{T}^{\prime}$.

Proof. The positivity of (5.3) for $q \in \mathbb{R}^{\prime}$ follows from the fact $[m]_{q^{2}}>0$ for $m \in \mathbb{Z}>0$. Let $q$ belong to the set $\mathbb{T}^{\prime}$. We first note that the form is positive definite if and only if $[m]_{q^{2}}>0$ for all $m>0$. Let $\hbar$ be the number such that $q^{2}=\exp \sqrt{-1} \hbar$ and $0<\hbar<2 \pi$. Then we have

$$
[m]_{q^{2}}=\frac{\sin (m \hbar)}{\sin \hbar}=\frac{\sin (m(2 \pi-\hbar))}{\sin (2 \pi-\hbar)} .
$$

Hence it suffices to discuss the case $0<\hbar<\pi$. Since $2<2 \pi / \hbar$, there exist a positive integer $m$ such that $m<2 \pi / \hbar \leqq m+1<2 m$. Then $[m]_{q^{2}}<0$, hence the form is not positive definite.

Proposition 5.3. Suppose $q^{4}$ is a primitive $L^{\text {th }}$ root of unity. Then there is a unique Hermitian form $(\mid)$ on $V_{\mathbf{p}}^{-}\left(\mathbf{p} \in \mathbb{Z}_{\geq 0}^{N}\right)$ such that $\left(x_{\mathbf{p}}(\mathbf{0}) \mid x_{\mathbf{p}}(\mathbf{0})\right)=1$ and $\left(V_{\mathbf{p}}^{-},(\mid)\right)$is a $*$-representation of $\mathscr{A}_{q}^{-}$. Explicitly the form $(\mid)$is given by

$$
\left(x_{\mathbf{p}}(\mathbf{m}) \mid x_{\mathbf{p}}(\mathbf{n})\right)=\delta_{\mathbf{m}, \mathbf{n}} q^{2 L \sum_{i} m_{i} p_{i}}\left[m_{1}\right] !_{q^{2}}\left[m_{2}\right] !_{q^{2}} \cdots\left[m_{N}\right] !_{q^{2}}, \quad\left(\mathbf{m}, \mathbf{n} \in\{0, \ldots, L-1\}^{N}\right) .
$$

The representation $\left(V_{\mathbf{p}}^{-},(\mid)\right)$is unitary if and only if $\mathbf{p} \in 2 \mathbb{Z}_{\geqq 0}^{N}$ and $q= \pm \exp ( \pm \sqrt{-1}(\pi / 2 L))$.

Proof. First we note that the form (|) is positive definite if and only if $q^{2 L p_{i}} \geqq 0$ $(1 \leqq i \leqq N)$ and $[m]_{q^{2}}>0$ for $m \in\{0, \ldots, L-1\}$. Let $k$ be the integer such that $q^{2}=\exp (\sqrt{-1} k \pi / L)$ and $1 \leqq k<L, L+1 \leqq k<2 L$. If $k=1$, then, for $1 \leqq m \leqq L-1$, we have $0<k m \pi / L<\pi$. Hence $[m]_{q^{2}}>0$ and the form is positive definite if and only if $\mathbf{p} \in 2 \mathbb{Z}_{\geq 00}^{N}$. Suppose $2 \leqq k \leqq L-1$. Since $L / k>1$, there exists an integer $m$ such that $1<L / k \leqq m<2 L / k \leqq L$. Then $[m]_{q^{2}}<0$, hence the form is not positive definite. The case $L+1 \leqq k<2 L$ is similar.

5.3. Unitary Representations of Enveloping Algebras. Now fix a real number $q \neq \pm 1$. We will define $*$-operations on quantum enveloping algebras by

$$
\left(e_{i}\right)^{*}=f_{i}, \quad\left(f_{i}\right)^{*}=e_{i}, \quad\left(k_{i}\right)^{*}=k_{i}
$$

for $U_{q}\left(A_{N-1}\right), U_{q}\left(B_{N}\right)$ and $U_{q}\left(D_{N}\right)$, and

$$
\left(e_{i}\right)^{*}=(-1)^{\delta_{i, N}} f_{i}, \quad\left(f_{i}\right)^{*}=(-1)^{\delta_{1, N}} e_{i}, \quad\left(k_{i}\right)^{*}=k_{i}
$$

for $U_{q}\left(C_{N}\right)$. We denote the corresponding *-algebras by $U_{q}(\mathfrak{s u}(N+1)), U_{q}(\mathfrak{s o}(2 N+1))$, $U_{q}(\mathfrak{s p}(2 N))$ and $U_{q}(\mathfrak{s p}(N, \mathbb{R}))$ respectively. Note that the map $*$ is a coalgebra 
automorphism, that is, if $\Delta(a)=\sum_{i} b_{i} \otimes c_{i}$ for $a \in U_{q}(X)$ then $\Delta\left(a^{*}\right)=\sum_{i} b_{i}^{*} \otimes c_{i}^{*}$. Hence the tensor product of two unitary representations is also unitary.

Proposition 5.4. Let the parameter $q$ belong to $\mathbb{R}^{\prime}$. Then, (1) The maps $\pi_{A}^{ \pm}, \pi_{B}^{+}, \pi_{C}^{-}$ and $\pi_{D}^{+}$are *-homomorphisms from $U_{q}(\mathfrak{s u}(N)), U_{q}(\mathfrak{s v}(2 N+1)), U_{q}(\mathfrak{s p}(N, \mathbb{R}))$ and $U_{q}(\mathfrak{s o}(2 N))$ to $\mathscr{A}_{q}^{ \pm}(N), \mathscr{A}_{q^{2}}^{+}(N), \mathscr{A}_{q}^{-}(N)$ and $\mathscr{A}_{q}^{+}(N)$ respectively.

(2) The module $V^{+}$is unitary as a representation of $U_{q}(\mathfrak{s u}(N)), U_{q}(\mathfrak{s p}(2 N+1))$ or $U_{q}(\mathfrak{s o}(2 N))$. The module $V^{-}$is unitary as a representation of $U_{q}(\mathfrak{s u}(N))$ or $U_{q}(\mathfrak{s p}(N, \mathbb{R}))$.

Proof. Part (1) is trivial. Part (2) immediately follows from Propositions 5.1 and 5.2 .

Note. Let the parameter $q$ belong to $\mathbb{T}^{\prime}$. Again there exist $*$-operations on quantum enveloping algebras such that the maps $\pi_{A}^{ \pm}, \pi_{B}^{+}, \pi_{C}^{-}$and $\pi_{D}^{+}$are $*$-algebra homomorphisms. These are defined by

$$
\left(e_{i}\right)^{*}=f_{i}, \quad\left(f_{i}\right)^{*}=e_{i}, \quad\left(k_{i}\right)^{*}=k_{i}^{-1}
$$

for $X=A_{N-1}, B_{N}, D_{N}$, and

$$
\left(e_{i}\right)^{*}=(-1)^{\delta_{i, N}} f_{i}, \quad\left(f_{i}\right)^{*}=(-1)^{\delta_{i, N}} e_{i}, \quad\left(k_{i}\right)^{*}=k_{i}^{-1}
$$

for $X=C_{N}$. By Propositions 5.1 and 5.3, the module $V^{+}$for $q \in \mathbb{T}^{\prime}$ and the module $V_{0}^{-}$for $q= \pm \exp ( \pm \sqrt{-1} \pi / 2 L)$ are unitary representations of the quantum enveloping algebras with respect to these *-operations. Unfortunately these *-operations are not coalgebra automorphisms but anti-coalgebra automorphisms, that is, if $\Delta(a)=\sum_{i} b_{i} \otimes c_{i}$ for $a \in U_{q}(X)$, then $\Delta\left(a^{*}\right)=\sum_{i} c_{i}^{*} \otimes b_{i}^{*}$.

\section{Spinor and Oscillator Representations of $U_{q}\left(A_{N-1}^{(1)}\right)$}

6.1. $\mathscr{A}_{q}^{ \pm}(\infty)$ and $U_{q}\left(A_{\infty}\right)$. In this section, we construct representations of the quantum enveloping algebra corresponding to the affine Cartan matrix $A_{N-1}^{(1)}$ which is given as follows:

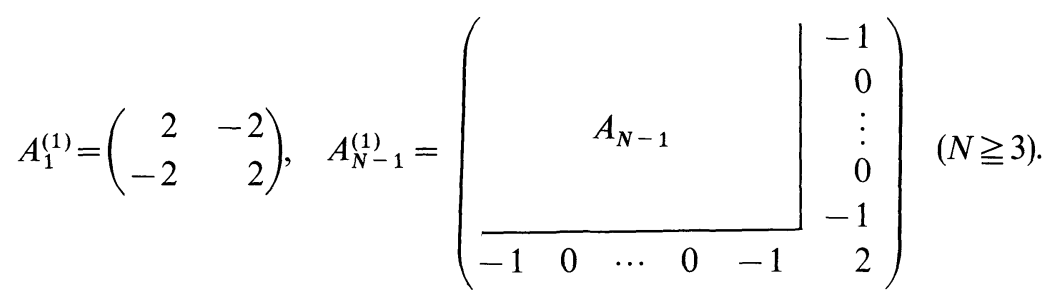

Let $\mathscr{A}_{q}^{ \pm}(\infty)$ be the algebra with generators $\left\{1, \psi_{i}, \psi_{i}^{\dagger}, \omega_{i}^{ \pm 1} \mid i \in \mathbb{Z}\right\}$ satisfying the defining relations $(2 \cdot 1)-(2 \cdot 5)$. Let $V^{ \pm}(\infty)$ be the linear span of the set

$$
\begin{aligned}
& I^{+}=\left\{\mathbf{m}=\left(m_{i}\right)_{i \in \mathbb{Z}} \mid m_{i} \in\{0,1\}(i \in \mathbb{Z}), m_{i}=0 \text { for }|i| \gg 0\right\}, \\
& I^{+}=\left\{\mathbf{m}=\left(m_{i}\right)_{i \in \mathbb{Z}} \mid m_{i} \in \mathbb{Z} \geqq 0(i \in \mathbb{Z}), m_{i}=0 \text { for }|i| \gg 0\right\} .
\end{aligned}
$$


We define the action of $\mathscr{A}_{q}^{ \pm}(\infty)$ on $V^{ \pm}(\infty)$ by the following; for any $\mathbf{m} \in I^{ \pm}$,

$$
\begin{aligned}
& \omega_{i} x(\mathbf{m})=\left\{\begin{array}{ll}
q^{-m_{l}} x(\mathbf{m}) & (i<0) \\
q^{m_{2}-1} x(\mathbf{m}) & (i \geqq 0)
\end{array},\right. \\
& \psi_{i} x(\mathbf{m})= \begin{cases}(-1)^{\sum_{j}<i} m_{j} x\left(\mathbf{m}-\mathbf{e}_{i}\right) & (i<0) \\
(-1)^{\sum_{j<i} m_{j}} x\left(\mathbf{m}+\mathbf{e}_{i}\right) & (i \geqq 0)\end{cases} \\
& \psi_{i}^{\dagger} x(\mathbf{m})= \begin{cases}(-1)^{\sum_{j}<^{m} m_{j}} x\left(\mathbf{m}+\mathbf{e}_{i}\right) & (i<0) \\
(-1)^{\sum_{j<i} m_{j}} x\left(\mathbf{m}-\mathbf{e}_{i}\right) & (i \geqq 0)\end{cases}
\end{aligned}
$$

for $\mathscr{A}_{q}^{+}(\infty)$ and

$$
\begin{aligned}
& \omega_{i} x(\mathbf{m})=\left\{\begin{array}{lll}
q^{m_{i}} x(\mathbf{m}) & (i<0) \\
q^{-m_{i}-1} x(\mathbf{m}) & (i \geqq 0)
\end{array},\right. \\
& \psi_{i} x(\mathbf{m})=\left\{\begin{array}{ll}
{\left[m_{i}\right]_{q^{2}} x\left(\mathbf{m}-\mathbf{e}_{i}\right)} & (i<0) \\
x\left(\mathbf{m}+\mathbf{e}_{i}\right) & (i \geqq 0)
\end{array}, \quad \psi_{i}^{\dagger} x(\mathbf{m})= \begin{cases}x\left(\mathbf{m}+\mathbf{e}_{i}\right) & (i<0) \\
-\left[m_{i}\right]_{q^{2}} x\left(\mathbf{m}-\mathbf{e}_{i}\right) & (i \geqq 0)\end{cases} \right.
\end{aligned}
$$

for $\mathscr{A}_{q}^{-}(\infty)$. Here we set $\mathbf{e}_{i}=\left(\delta_{i j}\right)_{j \in \mathbb{Z}}$ and $x(\mathbf{n})=0$ for $\mathbf{n} \notin I^{ \pm}$. Let $U_{q}\left(A_{\infty}\right)$ be the Hopf algebra with generators $1, e_{i}, f_{i}, k_{i}^{ \pm 1}(i \in \mathbb{Z})$ satisfying the defining relations (1.1) (1.8) with respect to the infinite rank Cartan matrix $A_{\infty}$ :

$$
A_{\infty}=\left[\left(A_{\infty}\right)_{i j}\right]_{l, j \in \mathbb{Z},}, \quad\left(A_{\infty}\right)_{i j}=\left\{\begin{array}{rl}
2 & i=j \\
-1 & |i-j|=1 . \\
0 & |i-j| \geqq 2
\end{array}\right.
$$

Similarly to Theorem 3.2 , we introduce the algebra homomorphisms $\pi_{A, \infty}^{ \pm}: U_{q}\left(A_{\infty}\right) \rightarrow$ $\mathscr{A}_{q}^{ \pm}(\infty)$ defined by

$$
e_{i} \mapsto \psi_{i-1} \psi_{i}^{\dagger}, \quad f_{i} \mapsto \psi_{i} \psi_{i-1}^{\dagger}, \quad k_{i} \mapsto\left(\omega_{i-1} \omega_{i}^{-1}\right)^{ \pm 1} \quad(i \in \mathbb{Z}) .
$$

6.2. Construction of the Representations. For $N \geqq 2$, introduce the operators on $V^{ \pm}(\infty)$ :

$$
\begin{gathered}
E_{i}:=\pi_{A, \infty}^{ \pm}\left(e_{i}\right)\left(\prod_{r \geqq 1} \pi_{A, \infty}^{ \pm}\left(k_{i-N r} k_{i+N r}^{-1}\right)\right), \quad(i \in \mathbb{Z}), \\
F_{i}:=\pi_{A, \infty}^{ \pm}\left(f_{i}\right)\left(\prod_{r \geqq 1} \pi_{A, \infty}^{ \pm}\left(k_{i-N r} k_{i+N r}^{-1}\right)\right), \quad(i \in \mathbb{Z}), \\
\tilde{k}_{i}:=\prod_{s \in \mathbb{Z}} \pi_{A, \infty}^{ \pm}\left(k_{i+N s}\right), \quad \tilde{e}_{i}:=\sum_{s \in \mathbb{Z}} E_{i+N s}, \quad \tilde{f}_{i}:=\sum_{s \in \mathbb{Z}} F_{i+N s} \quad(0 \leqq i \leqq N-1) .
\end{gathered}
$$

These operators prove to be well defined. In fact, for each $\mathbf{m} \in I^{ \pm}$, there exists an integer $M$ such that

$$
\pi_{A, \infty}^{ \pm}\left(k_{i}\right) x(\mathbf{m})=x(\mathbf{m}), \quad \pi_{A, \infty}^{ \pm}\left(e_{i}\right) x(\mathbf{m})=0, \quad \pi_{A, \infty}^{ \pm}\left(f_{i}\right) x(\mathbf{m})=0
$$

for $|i| \geqq M$.

Theorem 6.1. There exists a representation of $U_{q}\left(A_{N-1}^{(1)}\right)$ on $V^{ \pm}(\infty)$ defined by $e_{i} \mapsto \tilde{e}_{i}$, $f_{i} \mapsto \tilde{f}_{i}$ and $k_{i} \mapsto \tilde{k}_{i}(0 \leqq i \leqq N-1)$. 
Proof. Here we give the proof only for the case $N \geqq 3$. First we prove the following identities in End $V^{ \pm}(\infty)$ :

$$
\begin{aligned}
& \tilde{k}_{i} E_{j} \tilde{k}_{i}^{-1}= \begin{cases}q^{2} E_{j} & i-j \equiv 0(\bmod N) \\
q^{-1} E_{j} & i-j \equiv \pm 1(\bmod N) \\
E_{j} & \text { otherwise }\end{cases} \\
& \tilde{k}_{i} F_{j} \tilde{k}_{i}^{-1}= \begin{cases}q^{-2} F_{j} & i-j \equiv(\bmod N) \\
q F_{j}, & i-j \equiv \pm 1(\bmod N) \\
F_{j} & \text { otherwise }\end{cases} \\
& E_{i} F_{j}-F_{j} E_{i}=\frac{\delta_{i j}}{q^{2}-q^{-2}}\left(\left(\prod_{r \leqq 0} \pi_{A, \infty}^{ \pm}\left(k_{i+N r}^{2}\right)\right)\left(\prod_{r>0} \pi_{A, \infty}^{ \pm}\left(k_{i+N r}^{-2}\right)\right)\right. \\
& \left.-\left(\prod_{r<0} \pi_{A, \infty}^{ \pm}\left(k_{i+N r}^{2}\right)\right)\left(\prod_{r \geqq 0} \pi_{A, \infty}^{ \pm}\left(k_{i+N r}^{-2}\right)\right)\right), \\
& {\left[E_{i}, E_{j}\right]=0,\left[F_{i}, F_{j}\right]=0 \quad(i-j \neq 0, \pm 1(\bmod N)),} \\
& E_{i}^{2} E_{j}-\left(q^{2}+q^{-2}\right) E_{i} E_{j} E_{i}+E_{j} E_{i}^{2}=0 \quad(i-j \equiv \pm 1(\bmod N)), \\
& F_{i}^{2} F_{j}-\left(q^{2}+q^{-2}\right) F_{i} F_{j} F_{i}+F_{j} F_{i}^{2}=0 \quad(i-j \equiv \pm 1(\bmod N)) .
\end{aligned}
$$

Fix an index $\mathbf{m} \in I^{ \pm}$and take an integer $R$ such that

$$
\begin{aligned}
\pi_{A, \infty}^{ \pm}\left(k_{i+N r}\right) x(\mathbf{m}) & =\pi_{A, \infty}^{ \pm}\left(k_{j+N r}\right) x(\mathbf{m})=x(\mathbf{m}), \\
E_{i+N r} x(\mathbf{m}) & =E_{j+N r} x(\mathbf{m})=F_{i+N r} x(\mathbf{m})=F_{j+N r} x(\mathbf{m})=0, \\
{\left[\pi_{A, \infty}^{ \pm}\left(k_{i+N r}\right), E_{j}\right] } & =\left[\pi_{A, \infty}^{ \pm}\left(k_{i+N r}\right), F_{j}\right]=\left[\pi_{A, \infty}^{ \pm}\left(k_{j+N r}\right), E_{i}\right] \\
& =\left[\pi_{A, \infty}^{ \pm}\left(k_{j+N r}\right), F_{i}\right]=0,
\end{aligned}
$$

for any integer $r$ satisfying $|r| \geqq R$. Then,

$$
\begin{aligned}
\left(E_{i} F_{j}-F_{j} E_{i}\right) x(\mathbf{m})= & \prod_{1 \leqq r \leqq R} q^{\left.-\left(A_{\infty}\right)_{l}\right\lrcorner_{N r, j}+\left(A_{\infty}\right)_{i+N r, j}} \pi_{A, \infty}^{ \pm}\left(e_{i} f_{j}-f_{j} e_{i}\right) K^{2} x(\mathbf{m}) \\
= & \frac{1}{q^{2}-q^{-2}} \delta_{i j} \pi_{A, \infty}^{ \pm}\left(k_{i}^{2}-k_{i}^{-2}\right) K^{2} x(\mathbf{m}) \\
= & \frac{\delta_{i j}}{q^{2}-q^{-2}}\left(\left(\prod_{r \leqq 0} \pi_{A, \infty}^{ \pm}\left(k_{i+N r}^{2}\right)\right)\left(\prod_{r>0} \pi_{A, \infty}^{ \pm}\left(k_{i+N r}^{-2}\right)\right)\right. \\
& \left.-\left(\prod_{r<0} \pi_{A, \infty}^{ \pm}\left(k_{i+N r}^{2}\right)\right)\left(\prod_{r \geqq 0} \pi_{A, \infty}^{ \pm}\left(k_{i+N r}^{-2}\right)\right)\right) x(\mathbf{m}),
\end{aligned}
$$

where $K=\prod_{1 \leqq r \leqq R} \pi_{A, \infty}^{ \pm}\left(k_{i-N r} k_{i+N r}^{-1}\right)$. This proves (6.10). Next, we show the relation (6.12) for two typical case; $j-i \in 1+N \mathbb{Z}_{>0}$ and $j=i+1$. If $j-i \in 1+N \mathbb{Z}_{>0}$, then, for $u=x(\mathbf{m}), E_{i} x(\mathbf{m})$,

$$
q^{-1} E_{i} E_{j} u=\pi_{A, \infty}^{ \pm}\left(e_{i} e_{j}\right) K^{2} u=\pi_{A, \infty}^{ \pm}\left(e_{j} e_{i}\right) K^{2} u=q E_{j} E_{i} u .
$$


Hence,

$$
\begin{aligned}
\left(E_{i}^{2} E_{j}-\left(q^{2}+q^{-2}\right) E_{i} E_{j} E_{i}+E_{j} E_{i}^{2}\right) x(\mathbf{m})= & E_{i}\left(E_{i} E_{j}-q^{2} E_{j} E_{i}\right) x(\mathbf{m}) \\
& +q^{-2}\left(E_{i} E_{j}-q^{2} E_{j} E_{i}\right) E_{i} x(\mathbf{m})=0 .
\end{aligned}
$$

If $j=i+1$, then

$$
\begin{aligned}
& E_{i} E_{i} E_{j} x(\mathbf{m})=\pi_{A, \infty}^{ \pm}\left(e_{i} e_{i} e_{j}\right) K^{3} x(\mathbf{m}) \\
& E_{i} E_{j} E_{i} x(\mathbf{m})=\pi_{A, \infty}^{ \pm}\left(e_{i} e_{j} e_{i}\right) K^{3} x(\mathbf{m}), \quad E_{j} E_{i} E_{i} x(\mathbf{m})=\pi_{A, \infty}^{ \pm}\left(e_{j} e_{i} e_{i}\right) K^{3} x(\mathbf{m}) .
\end{aligned}
$$

Hence (6.12) follows from the relation (1.4) for the algebra $U_{q}\left(A_{\infty}\right)$. The remaining parts of (6.9)-(6.13) are similarly obtained. Now we show the operators $\tilde{e}_{i}, \tilde{f}_{i}, \widetilde{k}_{i}$ satisfy the relation (1.3). Let $S \geqq R$ be an integer such that $\left[E_{i+N s}, F_{j+N t}\right]=$ $\left[E_{i+N t}, F_{j+N s}\right]=0$ for $|t| \leqq R$ and $|s| \geqq S$. Then

$$
\begin{aligned}
{\left[\tilde{e}_{i}, \tilde{f}_{j}\right] x(\mathbf{m})=} & \sum_{-S \leqq s, t \leqq S}\left[E_{i+N s}, F_{j+N t}\right] x(\mathbf{m}) \\
= & \frac{\delta_{i j}}{q^{2}-q^{-2}}\left(\left(\prod_{r \leqq 0} \pi_{A, \infty}^{ \pm}\left(k_{i+N S+N r}^{2}\right)\right)\left(\prod_{r>0} \pi_{A, \infty}^{ \pm}\left(k_{i+N S+N r}^{-2}\right)\right)\right. \\
& \left.-\left(\prod_{r<0} \pi_{A, \infty}^{ \pm}\left(k_{i-N S+N r}^{2}\right)\right)\left(\prod_{r \leqq 0} \pi_{A, \infty}^{ \pm}\left(k_{i-N S+N r}^{-2}\right)\right)\right) x(\mathbf{m}) \\
= & \frac{\delta_{i j}}{q^{2}-q^{-2}}\left(\tilde{k}_{i}^{2}-\tilde{k}_{i}^{-2}\right) x(\mathbf{m})
\end{aligned}
$$

as required. The other relations of (1.1)-(1.5) follow clearly from (6.9)-(6.13). Thus we get the theorem.

Note. In $[\mathrm{FJ}]$ and $[\mathrm{B}]$, the vertex operator representations of quantum affine algebras are constructed. The relation between the vertex and the spinor representations of quantum affine algebras will be studied elsewhere.

Acknowledgements. The author would like to express his thanks to Prof. A. Tsuchiya for suggesting to him the importance of the $q$-analogue. He also wishes to express his thanks to Prof. Y. Kanie and Prof. K. Hasegawa for valuable discussions, and to Prof. E. Date, Prof. M. Jimbo, Prof. T. Miwa and Prof. M. Okado for their encouragement and interest in the work.

\section{References}

[B] Bernard, D.: Vertex operator representations of the quantum affine algebra $U_{q}\left(B_{r}^{(1)}\right)$, preprint 1988

[D] Drinfeld, V. G.: Quantum groups, ICM proceedings, pp. 798-820. New York: Berkley 1986

[DJKM] Date, E., Jimbo, M., Kashiwara, M. and Miwa, T.: Operator approach to the KadomtsevPetviashvili equation. Transformation groups for soliton equations. III. J. Phys. Soc. Jpn. 50, 3806-3812 (1981)

[DJM] Date, E., Jimbo, M., Miwa, T.: Representations of $U_{q}(\mathrm{gl}(n, \mathbb{C}))$ at $q=0$ and the RobinsonShensted correspondence, preprint 1989

[FF] Feingold, A. J., Frenkel, I. B.: Classical affine algebras. Adv. Math. 56, 117-172 (1985)

[FJ] Frenkel, I. B., Jing, N.: Vertex representations of quantum affine algebras, preprint 1988 
[J1] Jimbo, M.: A $q$-difference analogue of $U(\mathrm{~g})$ and the Yang-Baxter equation. Lett. Math. Phys. 10, 63-69 (1985)

[J2] Jimbo, M.: A $q$-analogue of $U(\operatorname{gl}(N+1))$, Hecke algebra and the Yang-Baxter equation. Lett. Math. Phys. 11, 247-252 (1986)

[KRS] Kulish, P. P., Reshetikhin, N. Yu., Sklyanin, E.K.: Yang-Baxter equation and representation theory. I. Lett. Math. Phys. 5, 393-403 (1981)

[L1] Lusztig, G.: Quantum deformation of certain simple modules over enveloping algebras, Adv. Math. 70, 237-249 (1988)

[L2] Lusztig, G.: Modular representations and quantum groups, preprint 1988

[M] Morikawa, H.: $\zeta_{n}$-Weyl algebra $W_{r}\left(\zeta_{n}, \mathbb{Z}\right)$. Nagoya Math. J. 113, 153-159 (1989)

[R] Rosso, M.: Finite dimensional representations of the quantum analog of the enveloping algebra of a complex simple Lie algebra. Commun. Math. Phys. 117, 581-593 (1988)

Communicated by $\mathrm{H}$. Araki

Received May 27, 1989

Note added in proof. After this paper has been submitted, Prof. Mitsuhiro Takeuchi of Tsukuba University Pointed out to the author that there exists an algebra isomorphism $A$ from $\mathscr{A}_{q}^{+}(N)$ to the $4 N$-fold direct product of the usual Clifford algebra $C(N):=\left\langle z_{i}\right| z_{i} z_{j}+z_{j} z_{i}=z_{i}^{\dagger} z_{j}^{\dagger}+z_{j}^{\dagger} z_{i}^{\dagger}=$ $\left.z_{i} z_{j}^{\dagger}+z_{j}^{\dagger} z_{i}-\delta_{i j}=0\right\rangle$. Let $I_{4}:=\{ \pm 1, \pm \sqrt{-1}\}$. For $\zeta=\left(\zeta_{i}\right) \in I_{4}^{N}$, we define an algebra map $\alpha_{\zeta}: \mathscr{A}_{q}^{+}(N) \rightarrow C(N)$ by $\alpha_{\zeta}\left(\psi_{i}\right)=\zeta_{i} z_{i}, \alpha\left(\psi_{l}^{\dagger}\right)=\zeta_{i} z_{l}^{\dagger}, \alpha\left(\omega_{i}\right)=\zeta_{i}\left(z_{i} z_{l}^{\dagger}+q^{-1} z_{l}^{\dagger} z_{i}\right)$. Then the map $A$ is given by $A(u)=\left(\alpha_{\zeta}(u)\right)\left(u \in \mathscr{A}_{q}^{+}(N)\right)$. The author is grateful to Prof. M. Takeuchi for this comment. 